\title{
La dose reçue par les patients au cours des examens de radiodiagnostic et son optimisation
}

C. MACCIA *

(Manuscrit reçu le 11 mai 1989)

RÉSUMÉ En 1988, à l'initiative de ta Commission des Communautés européennes, un groupe d'experts européens a réalisé une étude visant à optimiser l'information diagnostique contenue dans un cliché radiographique ainsi que l'irradiation des patients soumis aux examens de radiodiagnostic. Dans ce cadre, une enquête a été effectuée auprès de 900 patients examinés dans 24 services de radiologie en Europe et des données ont été recueillies pour les examens radiologiques suivants: thorax, crâne, colonne lombaire, bassin, appareil urinaire et sein. Les principaux résultats de cette enquête sont présentés. Les techniques radiologiques utilisées au cours de ces examens ainsi que les doses à l'entrée mesurées directement sur les patients sont analysées. Sur la base d'une "note médicale" définie par un groupe de radiologues, chaque incidence radiologique a été évaluée en vue de rechercher la technique d'examen "optimale". Une telle approche a nécessité la prise en compte des différents paramètres physiques qui influencent les doses reçues par les patients et la qualité des images radiologiques ( $\mathrm{kVp}$, exposemètre àutomatique, classe de sensibilité des couples film-écran....).

ABSTRACT In 1988, a study group of the Radiation protection programme of the CEC took the initiative of setting up a project which aimed at optimizing both the radiographic diagnostic information and the patient exposures associated to the radiological examinations. In this context a trial was conducted on some 900 patients examined at 24 different European x-ray departments and data were gathered on the following six common types of $x$-ray examination: chest, skull, lumbar spine, peivis and sacrum, urinary tract and breast. The main results obtained are presented. The radiological techniques used in performing these examinations throughout 10 European countries as well as the entrance skin doses directly measured on the patients are analyzed. On the basis of a "medical scoring system" defined by a group of radiologists, each radiographic projection was assessed in order to approach the "most efficient" way of performing the examination. This was carried out taking into account the most relevant physical parameters that may affect the dose received by the patient (kV, automatic exposure control system film-screen combinations...), as well as the quality of the radiographic image.

* CAATS - INSERM U. 240, 165, av. A.-Briand, 94230 Cachan. 


\section{INTRODUCTION}

L'exposition des populations aux rayonnements ionisants, résultant des applications industrielles et médicales de la radioactivité ainsi que des sources naturelles, fait l'objet d'une attention soutenue au niveau international. Le Comité scientifique des Nations Unies pour l'étude des effets des rayonnements ionisants (UNSCEAR) produit notamment, dans ses rapports successifs, des synthèses des données existantes sur les niveaux d'irradiation de toutes provenances et sur les effets biologiques correspondants [9]. Parmi ces différentes sources, les pratiques de radiodiagnostic constituent, pour les pays industrialisés, le mode principal d'irradiation du public d'origine "non naturelle". Le développement continu des pratiques de diagnostic, l'extension des indications ainsi que l'augmentation du nombre d'actes (le nombre d'examens est ainsi passé en France de quelque 6,2 millions en 1957 à environ 55 millions en 1988 [3-6], le rythme très rapide des innovations technologiques en imagerie médicale (notamment, celles qui interviennent du point de vue de la réduction des doses: amplificateur de brillance, écran aux terres rares, scopie TV...) rendent nécessaire une réévaluation périodique de ces niveaux d'exposition. II est, par ailleurs, intéressant pour le médecin, de disposer d'indicateurs du risque potentiel associé aux examens qu'il prescrit ou qu'il exécute, afin d'être en mesure d'apprécier réellement leur caractère plus ou moins "invasif", dans un contexte de sensibilisation générale du public aux problèmes d'irradiation.

C'est ainsi que de nombreuses études ont été réalisées dans certains pays européens tels que la Grande-Bretagne [11], l'Italie [10] et la France [9] en vue d'évaluer et de rationaliser l'utilisation médicale des radiations ionisantes dans le domaine du radiodiagnostic. Ces études, essentiellement à caractère technique (mesure de la dose et des paramètres susceptibles de l'influencer) ont ainsi montré une très grande variabilité des doses reçues par les patients, tant au niveau d'un même type d'examen, qu'en fonction de la technique d'imagerie utilisée. Toutes, elles constituent, malgré l'absence de tout renseignement concernant la qualité des images radiographiques obtenues au cours des examens étudiés, une importante base de données, point de départ indispensable pour des considérations d'évaluation et d'optimisation des pratiques radiologiques.

A travers l'unique indicateur physique que représente la dose reçue par le patient, on peut, en effet, essayer d'apprécier la qualité d'une image radiographique et, par conséquent, la qualité de l'information diagnostique qu'elle contient. Cependant, une telle approche n'est pas suffisamment pertinente et fiable pour tenter de rechercher la meilleure "qualité d'image" envisageable pour un cliché donné en fonction des caractéristiques du système de reproduction d'image utilisé (couple écran-film, sensibilité des détecteurs...). Un tel objectif ne peut être atteint que par la mesure directe des quantités physiques propres au système de reproduction telles que la densité optique du film, la "fonction de transfert de modulation" (FTM), la résolution spatiale..., ce qui, matériellement est difficilement réalisable au niveau d'un service de radiologie hospitalier. 
Outre ce type d'approche, de nature physique, il existe des méthodes d'évaluation de la qualité de l'image qui reposent exclusivement sur la capacité individuelle de l'observateur à visualiser des anomalies représentées sur un cliché radiographique (courbes ROC). Ces méthodes font appel d'une part à la perception subjective du contenu de l'image radiologique, ce qui relève plus de l'expérience de l'observateur que des paramètres techniques intrinsèques de l'image, et d'autre part elles privilégient l'indication diagnostique de l'examen (recherche d'images pathologiques).

Malheureusement, aucune de ces méthodes ne peut aisément s'appliquer dans la pratique sans l'existence d'un cadre méthodologique bien défini et sans disposer de moyens techniques considérables. Or, le radiologue est confronté, dans sa pratique médicale de tous les jours, au problème fondamental de l'évaluation de la qualité des images et de leur contenu diagnostic. A cela s'ajoute le choix de la technique radiologique la plus performante susceptible d'assurer des niveaux d'exposition pour les patients aussi faibles que possible.

Le respect de cette double contrainte, médicale et technique, conduit à la recherche du meilleur compromis "qualité d'image-dose d'irradiation".

C'est à ce but qu'un groupe d'experts européens, composé de radiologues et de physiciens, à l'initiative de la Direction générale XII de la Commission des Communautés européennes, s'est attaché en établissant, pour 6 examens de radiologie conventionnelle (thorax, crâne, colonne lombaire, bassin, appareil urinaire, sein), des listes de critères techniques, radiologiques et dosimétriques destinés à l'évaluation de la qualitè des images radiographiques obtenues au cours de ces examens.

L'objectif de cet article est de présenter d'une part, les résultats de l'enquête multinationale réalisée en 1988 dans dix pays européens, en vue d'évaluer la pertinence de ces critères et, d'autre part, de mener quelque réflexion au sujet de la réduction et de l'optimisation de la dose reçue par les patients au cours de ces examens.

\section{MÉTHODE}

Afin de pouvoir tester et valider sur un nombre d'examens radiologiques suffisamment important l'ensemble des critères définis par le groupe d'experts, une enquête multinationale a été réalisée auprès de plusieurs services de radiologie européens qui, spontanément, ont bien voulu participer à cette expérience. L'organisation de cette enquête a ainsi nécessité, outre la participation active du personnel médical et paramédical des différents services de radiologie contactés, la collaboration de différents centres de recherche et laboratoires responsables à la fois du suivi dosimétrique et de l'élaboration statistique des données.

Les données relatives à chaque type d'examen et à chaque cliché radiographique correspondant ont été collectées à l'aide d'un questionnaire dont les items concernaient, dans l'ordre: la technique radiologique, le patient, les critères d'image et la dose reçue par le patient. 


\section{a) La technique radiologique}

Pour un même type d'examen, les conditions techniques de prise des clichés ainsi que les caractéristiques de l'équipement radiogène utilisé peuvent sensiblement varier d'un service radiologique à un autre, jouant ainsi un rôle important sur la dose reçue par le patient. Aussi était-il nécessaire de recueillir des données précises concernant la filtration du tube radiogène, la taille du foyer, la grille antidiffusante, la classe de sensibilité des couples film-écrans radiologiques utilisés.

\section{b) Le patient}

Les données morphologiques des patients (âge, sexe, poids et taille) sont des éléments indispensables pour l'interprétation des résultats de l'examen radiologique et de la dosimétrie.

\section{c) Les critères d'image du cliché radiographique}

Apprécier la qualité de l'information médicale représentée dans un cliché radiographique n'est pas chose aisée. Cela dépend de facteurs morphologıques (corpulence du patient), techniques (incidence et positionnement du patient), physiques (qualité du rayonnement) et physiologiques (vision du radiologue) qui, tous ensemble, interagissent avec le résultat final de l'examen: le diagnostic.

Dans le contexte précis de l'enquête et compte tenu des objectifs de cette étude, les radiologues, membres du groupe d'experts européens, se sont donc efforcés de dresser des listes de critères d'image concernant les structures et les détails anatomiques qui devraient être toujours visualisés, ou visibles de manière satisfaisante, sur un cliché radiographique pour pouvoir le considérer comme acceptable pour le diagnostic. Volontairement, ces listes de critères radiologiques ont été établies de façon exhaustive et en dehors de toute considération pathologique.

Les examens radiologiques suivants ont été considérés : le thorax en incidence postéro-antérieure (P/A), le crâne en incidence antéro-postérieure $(A / P)$, (P/A) et de profil, le bassin de face $(A / P)$, la colonne lombaire en incidence $(A / P)$ et de profil, la charnière lombo-sacrée de profil, l'appareil urinaire en incidence (A/P) avant et après injection de produit de contraste et, enfin, le sein en incidence crânio-caudale. On trouvera, ci-dessous, un exemple des critères d'image ainsi définis par les radiologues et concernant le cliché du thorax en incidence (P/A):

- visibilité de six arcs costaux antérieurs,

- représentation symétrique du thorax,

- visibilité des limites costo-pleurales des apex pulmonaires au diaphragme,

- aspects des vaisseaux au niveau du poumon périphérique,

- nette reproduction des vaisseaux périphériques, du bord du cœur et du diaphragme,

- reproduction du poumon rétrocardiaque et du médiastin. 
La liste complète des critères d'image devant être vus sur les clichés considérés est fournie, en annexe.

\section{d) La dose recue par le patient}

Les problèmes méthodologiques posés par l'évaluation des doses et des risques associés à la pratique des examens de radiodiagnostic sont, de nos jours, loin encore d'être parfaitement résolus (choix de la quantité physique la plus pertinente à mesurer, type d'instrument de mesure, étalonnage des dosimètres...). Dans un double souci de faisabilité et de simplification, la méthode dosimétrique retenue ici a été l'utilisation de dosimètres thermoluminescents (DTL) positionnés directement sur les patients au cours des examens. Ainsi, pour chaque film et pour chaque incidence, un DTL, préalablement étalonné aux énergies caractéristiques du radiodiagnostic, a été placé, aussi près que possible du centre du champ d'irradiation, sur la peau du sujet examiné à la face d'entrée du rayonnement. De cette facon, l'équivalent de dose à l'entrée (incluant le rétrodiffusé) a pu être mesuré et relié aux paramètres techniques utilisés au cours de la prise des clichés.

\section{e) Détermination d'une valeur de dose de référence}

En vue de rechercher une relation entre la qualité de l'image radiographique et la dose reçue par le patient au cours d'un examen, une valeur de dose de référence a été établie pour chaque type de cliché considéré. Cette valéur a été déterminée, dans un premier temps, à partir des résultats obtenus au cours de l'évaluation de l'irradiation collective des patients soumis aux examens de radiodiagnostic en Grande-Bretagne [8]. Cette étude, réalisée entre 1983 et 1984 , fait état de mesures dosimétriques relatives à 3200 patients examinés dans 20 hôpitaux choisis áléatoirement parmi l'ensemble des établissements hospitaliers anglais. Pour tous les examens considérés ici, à l'exception de la mammographie, cette étude permet d'estimer des valeurs de dose à l'entrée représentatives de la pratique radiologique habituelle, c'est-à-dire d'une pratique d'examen conduisant à une qualité d'image pouvant être considérée comme satisfaisante pour le diagnostic. Les caractéristiques des données concernant les doses mesurées dans cette étude sont fournies dans le tableau I, en fonction du type d'incidence.

TABLEAU I

Caractéristiques des données concernant les doses à l'entrée observées dans l'étude anglaise de 1983 [8]

\begin{tabular}{|l|c|c|c|c|c|}
\hline \multirow{2}{*}{ Examens } & \multicolumn{5}{c|}{ Dose à l'entrée (mGy) } \\
\cline { 2 - 6 } & min. & $1^{\text {er }}$ percentile & Médiane & $3^{\text {e }}$ percentile & max. \\
\hline Thorax (P/A) & 0,03 & 0,13 & 0,18 & 0,26 & 1,43 \\
Crâne (P/A) & 1,82 & 3,26 & 4,25 & 5,49 & 13,1 \\
Crâne (A/P) & 0,73 & 2,97 & 4,02 & 4,97 & 13,9 \\
Crâne (profil) & 0,36 & 1,42 & 2,19 & 2,85 & 9,09 \\
Colonne lomb. (A/P) & 0,83 & 5,65 & 7,68 & 11,2 & 59,1 \\
Colonne lomb. (profil) & 2,38 & 12,7 & 19,7 & 30,1 & 108 \\
Charnière L5-S1 & 7,4 & 24 & 34,5 & 50,2 & 131 \\
Bassin (A/P) & 0,85 & 4,19 & 5,67 & 7,86 & 31,6 \\
Appareil urinaire (A/P) & 0,71 & 4,69 & 6,68 & 10,5 & 62,4 \\
\hline
\end{tabular}


Comme on peut le constater, quel que soit l'examen considéré, des écarts importants existent entre les valeurs de dose minimales et maximales, (facteur 50). D'autre part, certaines valeurs de doses ( $3^{e}$ percentile) laissent supposer, en absence de tout renseignement complémentaire concernant la sensibilité des systèmes de détection utilisés (couple film-écran), une qualité d'image insatisfaisante pour un bon diagnostic (noircissement du film trop important). D'autres valeurs en revanche ( $1^{\text {er }}$ percentile), traduisent, en principe, une qualité d'image insuffisante (films probablement trop clairs). Or, dans l'optique d'une détermination d'une valeur de référence de la dose qui ne serait pas à dépasser, afin d'éviter une dégradation trop importante de la qualité de l'image, l'analyse des données recueillies dans l'étude anglaise montre que, dans $14 \%$ des hôpitaux considérés, les doses étaient inférieures à la valeur correspondant au $1^{\text {er }}$ percentile de la distribution, dans $40 \%$ des cas, elles étaient inférieures à la valeur médiane ( $2^{\mathrm{e}}$ percentile) et enfin, dans $75 \%$ des cas, elles étaient inférieures au $3^{e}$ percentile.

Si l'on exclut donc les patients dont les doses dépassaient cette dernière valeur, 3/4 d'entre eux ont bénéficié d'un examen dont la qualité de l'image était tout à fait satisfaisante du point de vue radiologique. Un tel pourcentage apparait suffisamment significatif pour retenir, en toute première approximation, les valeurs de doses correspondantes aux $3^{\mathrm{e}}$ percentiles comme des valeurs de doses de référence. Ces valeurs sont résumées dans le tableau II.

TABLEAU ॥

Valeurs de référence des doses à l'entrée retenues à partir de l'étude anglaise [11]

\begin{tabular}{|l|c|}
\hline \multicolumn{1}{|c|}{ Examens } & Dose à l'entrée (mGy) \\
\hline Thorax (P/A) & 0,3 \\
Crâne (P/A) & 5 \\
Crâne (A/P) & 5 \\
Crâne (profil) & 3 \\
Colonne lombaire (A/P) & 10 \\
Colonne lombaire (profil) & 30 \\
Charnière L5-S1 & 50 \\
Bassin (A/P) & 10 \\
Appareil urinaire (A/P) & 10 \\
Sein (crânio-caudale) & 5 \\
\hline
\end{tabular}

En ce qui concerne la mammographie, la valeur de dose de référence retenue a été, dans un premier temps, celle déterminée expérimentalement par les Hollandais du Radiobiological institute (TNO) à partir d'un fantôme équivalent-tissu correspondant à un sein compressé de $5 \mathrm{~cm}$ d'épaisseur.

\subsection{Recueil des données}

Les données relatives aux différentes modalités et paramètres de chaque examen ont été recueillies selon un protocole détaillé dans le questionnaire reçu par les services de radiologie. Dix patients adultes de 
taille moyenne ont été ainsi considérés dans chacune des catégories d'examens étudiés. La technique de réalisation des clichés a été celle couramment employée dans le service de radiologie participant à l'enquête; en aucun, cas, des contrôles techniques préalables n'ont été effectués sur les installations radiologiques concernées (statifs, tubes, cassettes, machines à développer, négatoscopes...).

Avant la prise du cliché, un DTL a été placé sur la peau du patient au centre du faisceau incident, selon le protocole établi dans le questionnaire, et la lecture des clichés a été ensuite réalisée, en double aveugle, par deux radiologues. L'observance des différents critères de qualité a été ainsi rapportée dans les questionnaires, de même que l'appréciation de leur pertinence vis-à-vis du diagnostic final.

A la fin de la série des dix patients, les DTL ont été recueillis et envoyés pour leur lecture dans les trois laboratoires dosimétriques européens impliqués dans l'enquête, respectivement: le National radiological protection board en Angleterre, l'hôpital "Santa Maria della Misericordia" à Udine en Italie et le Gesellschaft für Strahlen- und Umweltforshung à Munich en Allemagne. Les résultats des lectures dosimétriques, conjointement aux questionnaires remplis, ont ensuite été transmis à l'Unité 240 de l'INSERM à Fontenay-aux-Roses (France) pour l'évaluation finale de l'enquête multinationale.

\section{RÉSULTATS}

\subsection{Généralités}

Vingt-quatre départements de radiologie de dix pays européens ont participé à l'enquête. Plus de 900 examens radiologiques ont été évalués et plus de 1200 mesures dosimétriques ont pu être effectuées directement sur les patients examinés. Le tableau III résume les résultats généraux relatifs à l'enquête.

TABLEAU III

Résultats généraux de l'enquête multinationale

\begin{tabular}{|l|c|c|c|c|}
\hline \multicolumn{1}{|c|}{ Examens } & $\begin{array}{c}\text { Nombre } \\
\text { de pays }\end{array}$ & $\begin{array}{c}\text { Nombre } \\
\text { de services }\end{array}$ & $\begin{array}{c}\text { Nombre } \\
\text { de patients }\end{array}$ & $\begin{array}{c}\text { Nombre de } \\
\text { dosimètres lus }\end{array}$ \\
\hline Thorax & 8 & 16 & 211 & 300 \\
Crâne & 5 & 12 & 117 & 223 \\
Colonne lombaire & 7 & 14 & 149 & 204 \\
Bassin & 6 & 13 & 139 & 134 \\
Appareil urinaire & 7 & 15 & 155 & 191 \\
Sein & 8 & 15 & 160 & 160 \\
\hline Total & 10 & 24 & 931 & 1212 \\
\hline
\end{tabular}


Chacun des examens a été pratiqué dans au moins 12 services de radiologie différents. Le nombre de patients ayant bénéficié des examens varie de 117 pour les clichés du crâne à 211 pour les radiographies thoraciques.

\subsection{Paramètres techniques utilisés}

Dans le tableau IV, sont résumées les valeurs minimales, moyennes et maximales des principaux paramètres techniques utilisés au cours du déroulement des examens considérés dans l'enquête. Une toute première remarque concerne le pourcentage élevé d'examens qui ont été pratiqués en présence de la grille antidiffusante. Ce dispositif, à défaut d'augmenter la dose reçue par le patient, permet d'obtenir une meilleure qualité d'image par rapport aux systèmes sans grille. Seuls $25 \%$ des clichés de mammographie font exception à cette tendance générale.

On constate, par ailleurs, que la majorité des clichés a été effectuée dans des conditions manuelles c'est-à-dire sans recours au système automatique de contrôle de l'exposition (cellule). Une telle pratique laisse supposer, de la part des utilisateurs, une sélection judicieuse du temps d'exposition et de l'intensité du courant en fonction des caractéristiques du couple écran-film et de la morphologie du patient examiné. II en découle une grande variabilité de ces paramètres et, en corollaire, une égale variabilité des doses et de la qualité de l'image. A ce titre, on peut mentionner l'étendue des temps d'exposition observés dans l'enquête, notamment dans le cas de la mammographie où environ $70 \%$ des examens ont été pratiqués manuellement et où ces temps varient, toute épaisseur de sein confondue, de $100 \mathrm{~ms}$ à $7500 \mathrm{~ms}$.

On peut enfin noter qu'en ce qui concerne la filtration totale des tubes, bien qu'il s'agisse de valeurs nominales et non pas mesurées par les utilisateurs, certains examens ont été pratiqués avec des filtrations inadaptées ( $\leq 1,5 \mathrm{~mm}$ d'Al) pour garantir une qualité d'image satisfaisante. En revanche, plus de $50 \%$ des clichés ont été réalisés dans des conditions de filtration totale correcte $(\geq 2,5 \mathrm{~mm}$ d'Al).

\subsection{Validation des critères d'image}

Le tableau $V$ indique, pour chaque incidence, les résutats concernant les taux de clichés nuls, les pourcentages de clichés pour lesquels les radiologues ont pu répondre affirmativement à tous les critères de qualité d'image et, enfin, les pourcentages de services de radiologie où, en moyenne, les doses mesurées sur les patients n'ont pas dépassé 
DOSE REÇUE AU COURS D'EXAMENS DE RADIODIAGNOSTIC ET SON OPTIMISATION

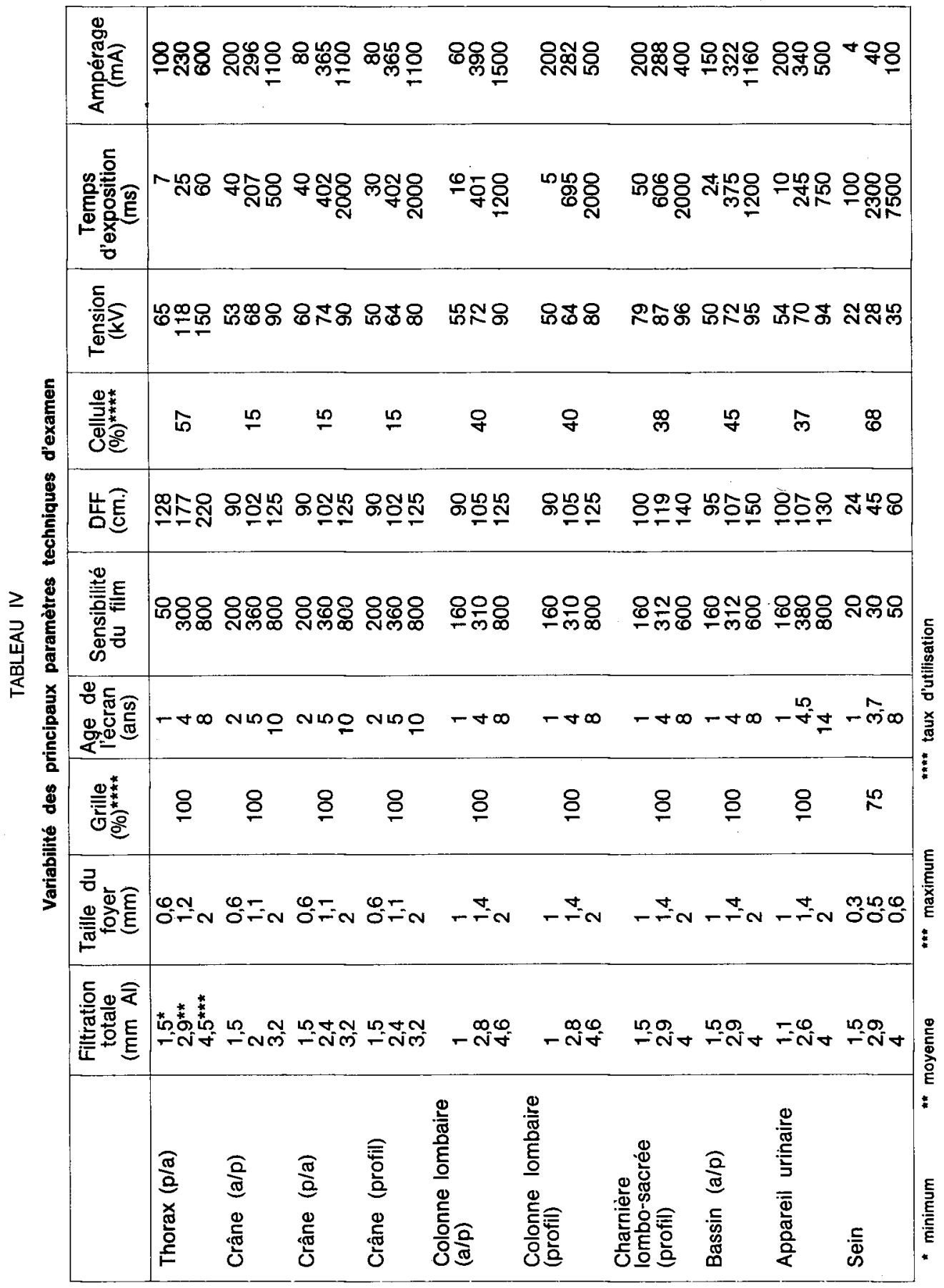


TABLEAU $V$

Indices de validité des résultats de l'enquête

\begin{tabular}{|l|c|c|c|}
\hline \multicolumn{1}{|c|}{ Examens } & $\begin{array}{c}\text { Clichés } \\
\text { nuls } \\
(\%)\end{array}$ & $\begin{array}{c}\text { Clichés } \\
\text { comportant } \\
\text { tous les critères } \\
(\%)\end{array}$ & $\begin{array}{c}\text { Services où la dose } \\
\text { de référence } \\
\text { a été respectée } \\
(\%)\end{array}$ \\
\hline Thorax (p/a) & 2 & 79 & 69 \\
Crâne (a/p) & 10 & 74 & 89 \\
Crâne (p/a) & 10 & 65 & 56 \\
Crâne (profil) & 5 & 67 & 75 \\
Colonne lombaire (a/p) & 10 & 69 & 50 \\
Colonne lombaire (profil) & 4 & 77 & 69 \\
Charnière L5-S1 (profil) & 5 & 83 & 67 \\
Bassin (a/p) & 6 & 77 & 58 \\
Appareil urinaire & 6 & 62 & 37 \\
Sein & 5 & 79 & 33 \\
\hline
\end{tabular}

la valeur de dose de référence. Tout d'abord, on observe que les taux de clichés nuls sont en accord avec ceux généralement rencontrés dans la littérature $[1,2,7,8]$, ce qui laisse supposer que la réalisation des clichés ainsi que leur lecture se sont effectuées dans des conditions de pratique habituelle. Deux examens (crâne et colonne lombaire) ont des taux de rejet égaux à $10 \%$, les autres, excepté le cliché thoracique (2\%), atteignent des pourcentages de clichés nuls compris entre 5 et $6 \%$. Bien que globalement satisfaisants quant à la démonstration de l'absence de biais méthodologiques dans l'enquête, ces taux, loin d'être négligeables, soulèvent, toutefois, le problème de la réduction du nombre de clichés inutiles et de la suppression d'une dose d'irradiation inutilement délivrée aux patients.

On constate, par ailleurs que, quel que soit l'examen envisagé, le pourcentage de clichés où tous les critères ont été vus est plutôt élevé ( $72 \%$ en moyenne). Cependant, si l'on ajoute à ces résultats les taux de clichés nuls correspondants, jamais le score de $100 \%$ n'est atteint. Cela signifie qu'un certain nombre de clichés. ont été jugés satisfaisants pour établir un diagnostic alors qu'ils ne possédaient pas tous les critères de qualité listés dans le questionnaire. En d'autres termes, les radiologues, dans leur pratique quotidienne, semblent moins restrictifs et portent un jugement moins sévère au sujet de la qualité des images radiographiques qu'ils produisent. $\mathrm{Ce}$ résultat pose donc le problème de l'adaptation des listes de critères à la pratique radiologique de tous les jours. Enfin, à partir de ces résultats, une relation a été recherchée entre la pratique de clichés de bonne qualité, réalisés logiquement dans des services à faible taux de clichés nuls, et leur niveau d"irradiation se traduisant par un pourcentage élevé d'hôpitaux travaillant en dessous des valeurs de doses de référence conseillées. Malheureusement, aucune corrélation statistiquement significative n'a pu être établie entre ces deux paramètres. Ainsi, aux clichés de crâne en incidence A/P réalisés dans $89 \%$ des services de radiologie dans des conditions d'irradiation correctes, correspond le taux de clichés nuls le plus 
élevé (10\%). En revanche, les clichés de thorax, caractérisés par un taux de clichés nuls très faible $(2 \%)$, aboutissent à des niveaux d'irradiation dépassant les niveaux de référence dans $31 \%$ des services de radiologie considérés.

\subsection{Le système de pondération des critères de qualité des clichés de radiodiagnostic}

Lors d'une première analyse des réponses aux questionnaires, il est apparu que certains clichés, jugés comme acceptables par les radiologues pour établir un diagnostic, ne présentaient pas, en même temps, tous les critères de qualité retenus. A titre d'exemple, la répartition des réponses aux critères de qualité pour le cliché du thorax est donnée dans le tableau VI:

TABLEAU VI

Répartition des réponses aux critères d'image (thorax P/A)

(effectif: 211 clichés)

\begin{tabular}{|c|c|c|}
\hline $\begin{array}{l}\text { CRITERES D'IMAGE } \\
\text { THORAX (P/A) }\end{array}$ & $\begin{array}{l}\text { OUI } \\
(\%)\end{array}$ & $\begin{array}{c}\text { NON } \\
(\%)\end{array}$ \\
\hline $\begin{array}{l}\text { - Six arcs costaux antérieurs visibles } \ldots \ldots \ldots \ldots \ldots \ldots \\
\text { - Thorax symétrique } \ldots \ldots \ldots \ldots \ldots \\
\text { - Limite costo-pleurale visible des apex pulmonaires au } \\
\text { diaphragme } \ldots \ldots \ldots \ldots \ldots \ldots \ldots \\
\text { - Aspect des vaisseaux au niveau du poumon périphérique } \\
\text { - Nette reproduction des vaisseaux périphériques, du bord } \\
\text { du cour et du diaphragme } \\
\text { - Poumon rétrocardiaque et médiastin } \ldots \ldots \ldots \ldots \ldots \ldots \ldots\end{array}$ & $\begin{array}{l}98,6 \\
98,6 \\
94,7 \\
98,1 \\
96,6 \\
88\end{array}$ & $\begin{array}{r}1,4 \\
1,4 \\
5,3 \\
1,9\end{array}$ \\
\hline - Acceptabilité du cliché pour le diagnostic & 99 & 1 \\
\hline
\end{tabular}

Comme on peut le remarquer, des différences sensibles existent dans les taux de réponses donnés par les radiologues: $12 \%$ des clichés thoraciques n'ont pas été jugés convenables pour la visualisation du poumon rétrocardiaque et du médiastin, tandis que $5,3 \%$ des clichés ne présentaient pas les limites costo-pleurales visibles des apex pulmonaires au diaphragme. Malgré cela, seulement $1 \%$ des clichés ont été jugés insuffisants pour une interprétation diagnostique. Ces résultats semblent donc en apparence discordants, mais ils peuvent s'interpréter par une inégalité d'importance accordée par les radiologues à chaque critère, lors de la lecture du cliché.

Afin de reproduire plus fidèlement cette démarche implicitement suivie par les radiologues, une étude complémentaire de ces réponses a été réalisée. Pour chaque critère d'image relatif à chaque examen, trois dimensions différentes ont été identifiées: une dimension médicale, une dimension technique, une dimension positionnelle. Ainsi, pour le cliché du thorax en incidence P/A et pour le critère "poumon rétrocardiaque et médiastin", la dimension médicale se traduit par le fait que cette région anatomique 
doit être impérativement visualisée sous peine de ne pas discerner une éventuelle pathologie. Or, cette visualisation ne peut être correcte que si le choix du kilovoltage est judicieusement réalisé et le contraste suffisant pour distinguer les structures de tonalité différente situées dans le thorax (dimension technique). Enfin, cette possibilité de visualiser ces éléments anatomiques dépend du centrage du rayon incident sur le patient (dimension positionnelle). Afin de pondérer l'importance relative de ces trois dimensions, tous les critères indiqués dans le questionnaire ont été soumis à l'appréciation d'un groupe de radiologues expérimentés qui leur a attribué des notes variant de 0 à 3 . A chaque critère correspond ainsi un "triplet". Lorsque la note est nulle, le critère est indifférent pour la dimension considérée, une note égale à 1 signifie que la dimension intervient peu dans le critère, une note égale à 2 signifie que la dimension est importante, enfin une note égale à 3 signifie que la dimension est fondamentale. A titre d'exemple, les notes concernant l'incidence P/A du thorax sont données dans le tableau VII.

TABLEAU VII

Exemple de notes attribueses par le groupe de radiologues (thorax P/A)

\begin{tabular}{|c|c|c|c|}
\hline \multirow{2}{*}{$\begin{array}{l}\text { CRITÈRES D'IMAGE } \\
\text { THORAX (P/A) }\end{array}$} & \multicolumn{3}{|c|}{ NOTES } \\
\hline & $T$ & $P$ & $M$ \\
\hline 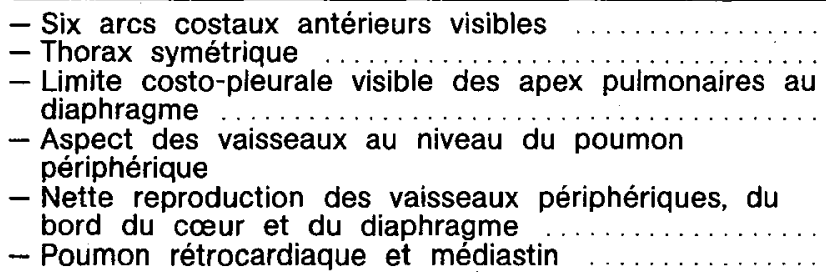 & $\begin{array}{l}3 \\
2\end{array}$ & $\begin{array}{l}2 \\
2\end{array}$ & $\begin{array}{l}2 \\
2\end{array}$ \\
\hline - Note globale & 14 & 7 & 12 \\
\hline
\end{tabular}

$T$ : Technique. $\mathrm{P}$ : Positionnement. $\mathrm{M}$ : Médical.

On peut ainsi noter que le même critère de qualité d'image peut ne pas avoir, pour le radiologue, la même importance si l'on considère le point de vue technique, médical ou positionnel.

Dans le cas particulier où le "triplet" est faible, on conçoit donc qu'une réponse négative au critère correspondant ait peu d'incidence sur l'acceptabilité du cliché. En d'autres termes, il est possible, pour le radiologue, de ne pas être en accord avec un critère d'image donné (réponse négative) sans pour autant juger le cliché inacceptable. On comprend alors mieux les réponses relatives au questionnaire qui, auparavant, paraissaient discordantes. Ce système de notes a été utilisé pour rechercher les relations entre les résultats dosimétriques et la qualité d'image des clichés radiographiques. Dans ce procédé, la note globale moyenne relative à chaque service de radiodiagnostic ayant participè à l'enquête a été choisie comme l'indicateur de qualité d'image le plus pertinent en l'absence de tout autre renseignement concernant les clichés (impossibilité de disposer des clichés 
directement). Cette valeur moyenne a été déterminée en tenant compte du nombre de clichés pratiqués dans chaque service et considérés comme acceptables pour le diagnostic.

\subsection{Evaluation des résultats}

Pour chaque incidence considérée, sont représentés, dans les figures 1 et 2, d'une part les doses moyennes calculees dans chaque service de radiologie et d'autre part les notes globales moyennes correspondantes.

La figure 1 montre que, en dépit de l'idée largement répandue que ce type d'incidence bénéficie d'une technique standardisée, les moyennes des doses mesurées dans les différents services de radiologie, graphiquement représentées en ordre croissant, varient de 0,16 à 0,95 mGy (facteur 6). En ce qui concerne la qualité des images radiographiques obtenues dans ces services, exprimée ici par les notes globales moyennes (courbe continue), il apparaît que, parmi les 16 services étudiés, deux seulement obtiennent la note globale moyenne maximale ( ${ }^{\circ s} 12$ et 14 ) mais au prix d'un dépassement de la dose de référence $(0,3 \mathrm{mGy})$. Par ailleurs, afin de visualiser la tendance générale de la qualité d'image, en fonction de l'augmentation de la dose dans chaque service, la droite de régression des notes a été tracée. II apparaît ainsi clairement que la tendance est à la réalisation de clichés dont la qualité d'image est comparable d'un hôpital à l'autre (pente de la droite de régression nulle), ce qui reflète, en première analyse, un souci de fiabilité et reproductibilité de la pratique des examens. Malheureusement cet objectif est parfois atteint, à cause d'une méconnaissance des doses correspondantes, au prix d'une irradiation injustifiée des patients.

Doses (mGy)

Note globale moyenne

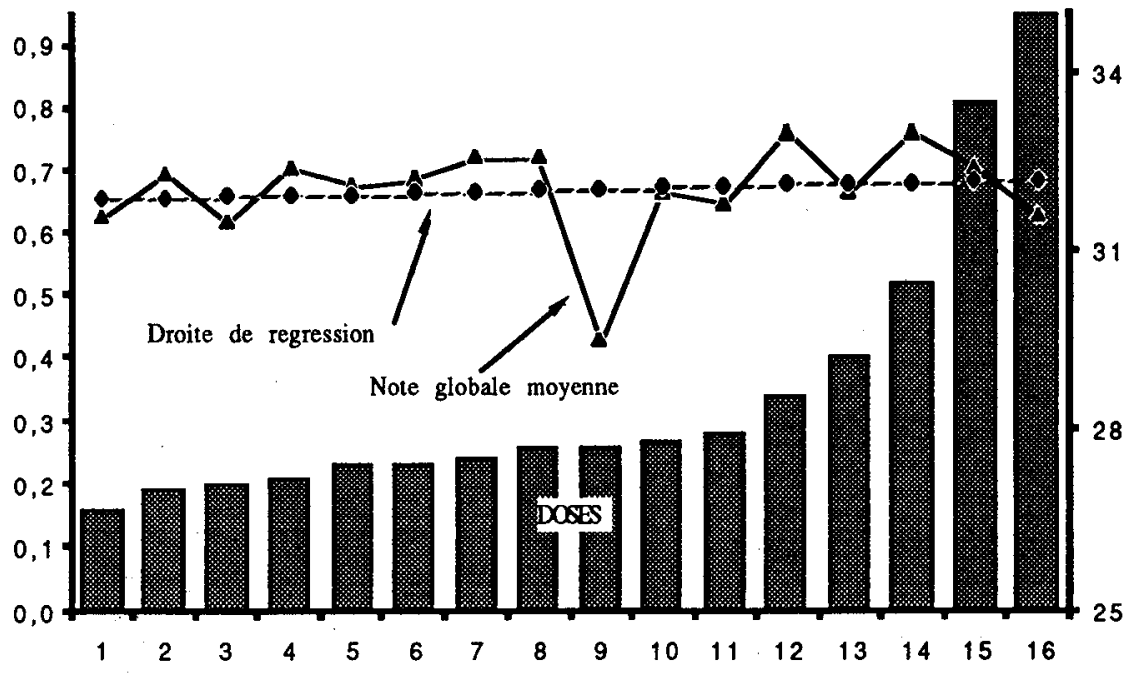

Fig. 1. - Histogramme des doses moyennes et notes globales moyennes par service de radiologie. Thorax (P/A). 
Bien que l'étendue des doses indiquées dans la figure 2 paraisse importante (de 2,5 à $30 \mathrm{mGy}$ ), seulement 2 services sur 15 dépassent la dose de référence de $10 \mathrm{mGy}$. Ici encore, le souci d'obtenir des clichés reproductibles et de bonne qualitè l'emporte sur celui de maintenir des doses à des niveaux acceptables (services $\mathrm{N}^{\text {os }} 14$ et 15 ). On peut, néanmoins, signaler que la majorité des clichés de bonne qualité (au-dessus de la droite de régression) est pratiquée avec des doses inférieures à $10 \mathrm{mGy}$.

\section{RECHERCHE DE LA TECHNIQUE OPTIMALE}

L'étape finale de l'évaluation de la pertinence des critères de qualité des clichés de radiodiagnostic a consisté à se servir du système de notes défini précédemment pour identifier des techniques radiologiques qui, à la fois, assureraient la meilleure information médicale et délivreraient aux patients des doses d'irradiation aussi faibles que possible. On a ainsi procédé à une sélection des clichés, incidence par incidence, uniquement en tenant compte de leur note médicale globale. La dimension médicale a été ainsi privilégiée par rapport aux autres dimensions initialement identifiées car elle correspond d'avantage à l'objectif poursuivi prioritairement lors de la réalisation d'un cliché radiologique.

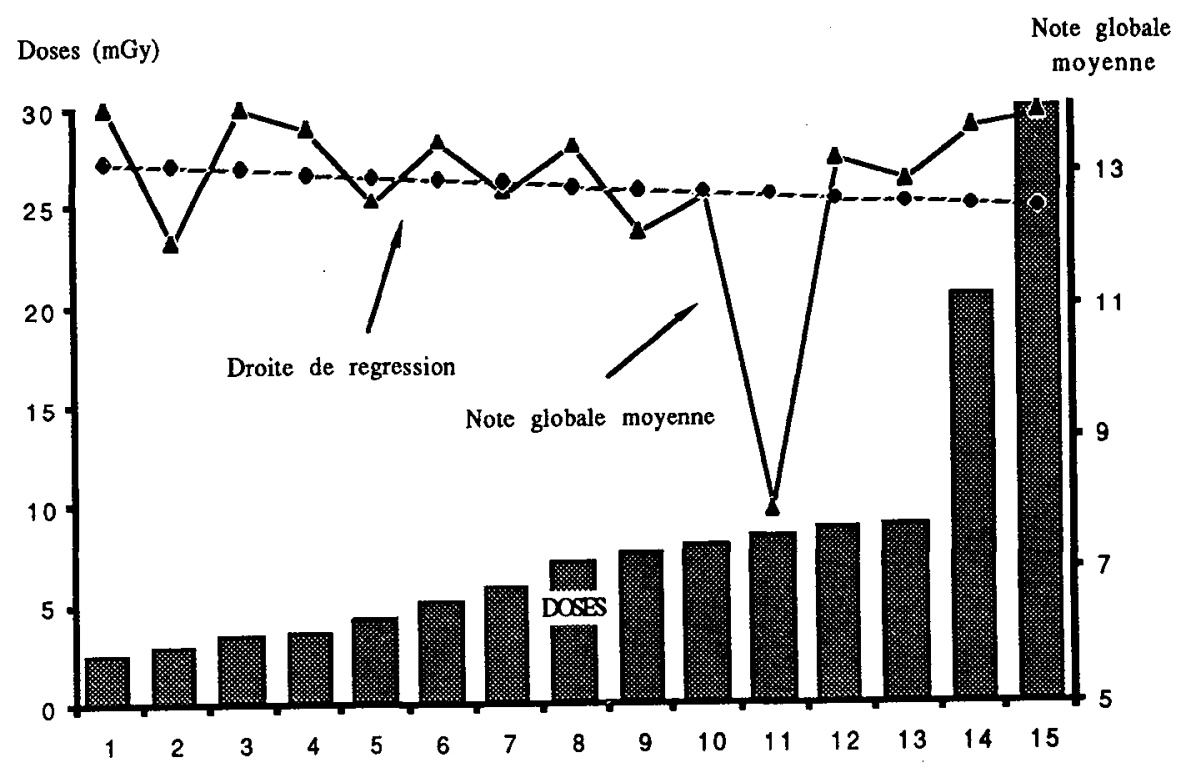

Fig. 2. - Histogramme des doses moyennes et notes globales moyennes par service de radiologie. Appareil urinaire (avant injection). 
Par ailleurs, afin de pouvoir illustrer les résultats de ce processus de sélection, les équipements radiologiques installés dans les différents services étudiés ont été répartis en quatre catégories. Ces catégories tiennent compte des caractéristiques des systèmes de détection d'image (sensibilité des couples film-écrans) ainsi que de leur mode de fonctionnement (manuel: choix manuel des paramètres d'exposition; automatique: cellule).

\subsection{Les techniques sélectionnées}

La mise en application de la procédure de sélection a permis d'effectuer une comparaison des différentes techniques radiologiques utilisées lors des incidences étudiées.

En moyenne, toute incidence confondue, $20 \%$ des clichés jugés satisfaisants pour établir un diagnostic ont été ainsi écartés à cause de leur mauvaise note globale médicale. Si l'on ajoute les clichés n'ayant pas respecté la contrainte supplémentaire concernant la valeur de dose de référence, ce pourcentage atteint alors environ $60 \%$ (59\% dans le cas du thorax et $49 \%$ dans le cas du cliché de l'appareil urinaire pratiqué avant injection du produit de contraste illustrés respectivement dans les figures 3 et 4$)$.

D'une manière générale, la plupart des clichés écartés ont été pratiqués avec des couples film-écrans de classe de sensibilité insuffisante et des techniques radiographiques au cours desquelles les paramètres physiques susceptibles d'influencer aussi bien la dose que l'image ont été déterminés manuellement. L'utilisation des exposemètres automatiques (cellule) associée à des couples film-écrans bien adaptés a, en revanche, fait preuve de tout son intérêt en assurant une moindre irradiation pour les patients à qualité d'image égale (note médicale globale identique). Enfin, lorsque la technique manuelle est utilisée correctement, les résultats sont aussi performants, voire meilleurs, que ceux obtenus avec la cellule (cas du cliché de l'appareil urinaire, par exemple).

Comme on peut le remarquer (fig. 3), parmi les 208 clichés jugés acceptables pour le diagnostic, 167 ont été retenus suite à la procédure de sélection. Trois techniques radiographiques ont été ainsi sélectionnées, toutes, équivalentes du point de vue de l'image. On constate tout d'abord l'absence d'examens pratiqués en mode manuel avec des systèmes de détection de faible classe de sensibilité $(<200)$, ce qui dénote une pertinence remarquable de la méthode de sélection utilisée (système de notes attribuées par les radiologues). 
En ce qui concerne les techniques mentionnées dans la figure 3 , la hiérarchie dosimétrique a été parfaitement respectée avec, en ordre croissant, les examens pratiqués en présence de la cellule et avec des couples écran-films dont la classe, de sensibilité est $\geq 200$, les examens pratiqués avec une technique manuelle et toujours des couples écran-films dont la classe de sensibilité est $\geq 200$, enfin, ceux qui ont été réalisés en présence de la cellule mais avec des systèmes de détection moins rapides $(<200)$. Pour ces derniers, d'ailleurs, la moyenne des doses mesurées sur les patients dépasse, dans tous les cas, la valeur de référence de 0,3 mGy.

Manuel $\geq 200$
Autom $\geq 200$
Autom $<200$

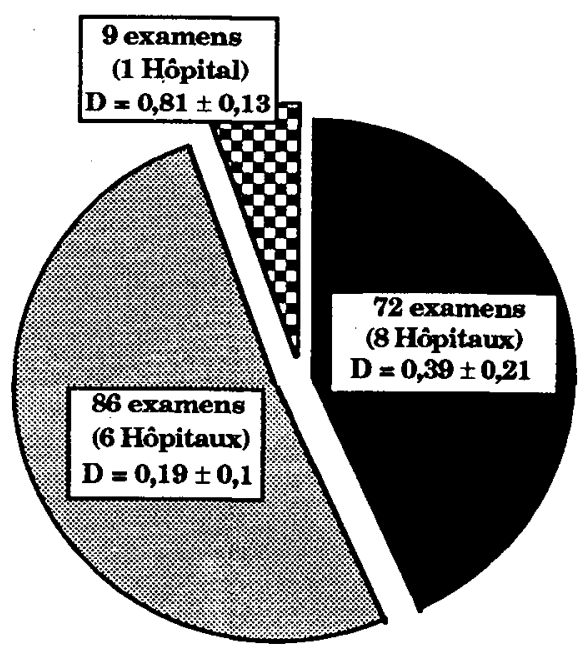

Fig. 3. - Répartition des clichés du thorax (P/A) résultant de la procédure de sélection sur la note médicale globale.

Ainsi, l'application de cette deuxième contrainte conduit à retenir d'une part, 86 clichés sur $208(59 \%)$ et, d'autre part, à ne considérer qu'une technique radiologique.

Parmi les trois catégories de techniques radiologiques sélectionnées (figure 4), deux semblent être parfaitement comparables tant du point de vue de la qualité de l'image que du point de vue dosimétrique "Autom $\geq 400$ " et "Manuel $\geq 400$ " respectivement. 


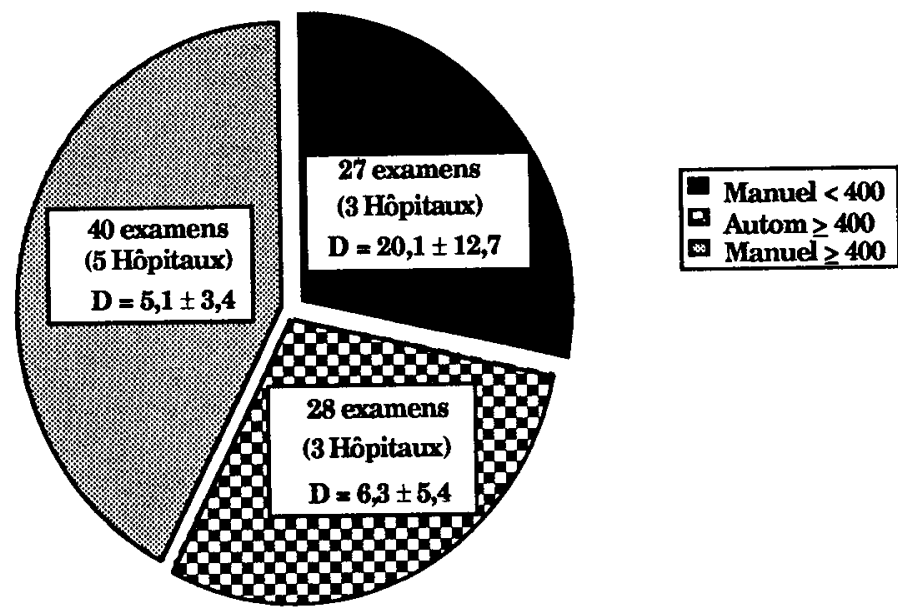

Fig. 4. - Répartition des clichés de l'appareil urinaire avant injection du produit de contraste résultant de la procédure de sélection sur la note médicale globale.

En moyenne, les doses délivrées au cours de ces examens sont inférieures aux valeurs de dose de référence. A la troisième catégorie appartiennent, par contre, des examens réalisés en mode manuel et à partir d'une mauvaise configuration de l'équipement destiné à la reproduction d'image c'est-à-dire des couples écran-films de faible classe de sensibilité "Manuel < 400". Toutes les doses correspondantes dépassent alors les niveaux de références. De telles situations ne peuvent aboutir qu'à des examens trop irradiants pour le patient qui, tout en bénéficiant du diagnostic, est soumis à une surexposition aux rayonnements totalement injustifiée.

\section{CONCLUSION}

L'analyse des résultats d'une enquête internationale réalisée dans 10 pays européens et concernant plus de 900 clichés de radiodiagnostic a contribué à la recherche du meilleur compromis "qualité d'image-dose" délivrée aux patients au cours de six types d'examens radiologiques conventionnels. L'approche suivie s'est révélée pertinente et elle a permis d'établir les modalités techniques "optimales" et de pratique radiologique répondant à cet objectif. 
Deux axes de recherche prioritaires vers lesquelles des efforts supplémentaires seraient à accomplir ont été identifiés :

1. La formation en radioprotection du personnel médical et paramédical pratiquant les examens (choix concerté des paramètres influençant l'irradiation des patients) ;

2. L'établissement de programmes d'assurance et de contrôle de qualité en radiodiagnostic (bonne utilisation des équipements radiologiques et réduction du nombre de clichés nuls).

Grâce à cette approche, qui utilise des moyens d'évaluation de la qualité de l'image et de la dose simples et applicables au niveau de chaque service de radiologie, il semble possible d'envisager une optimisation des pratiques radiologiques habituelles. Enfin, cette première étape du processus d'optimisation (amélioration de la qualité de l'image et réduction de l'irradiation délivrée au patient) souligne à la fois la nécessité et l'intérêt d'une collaboration accrue entre les radiologues et les physiciens dans les services de radiodiagnostic.

\section{RÉFÉRENCES}

[1] AMERICAN COLLEGE OF RADIOLOGY. Quality assurance in diagnostic radiology and nuclear medicine - the obvious decision. (HHS publication $N^{O}$ (FDA) 81-8141) Washington, D.C. : U.S. Department of Health and Human Services, 1981.

[2] BELLETTI R., GALLINI R., GIUGNI U. Operative programme of quality control in the radiodiagnostic field in a general hospital : results and proposals. B.J.R. Supplement $n^{\circ} 18,131-133$.

[3] CHARPAKY., MACCIA C., FAGNANI F. Etude prospective sur l'imagerie médicale en France. Rapport INSERM $n^{\circ} 51,1988$.

[4] FAGNANI F., THIERRY J.P., MACCIA C., LEFAURE C., BENEDITTINI M., MIZRAHI A., FAURE $H$. Enquête nationale sur le radiodiagnostic : Partie 1 : Aspects méthodologiques. J. Radiologie 66 (2) : 167-174; 1985.

[5] FAGNANI F., LEFAURE C., THIERRY J.P., MACCIA C., BENEDITTINI M., MIZRAHI A., FAURE H. Enquête nationale sur le radiodiagnostic : Partie 2 : Les moyens en personnel et en matériel. J. Radiologie $66(3): 245-251 ; 1985$.

[6] FAGNANI F., MACCIA C., LEFAURE C., BENEDITTINI M., MIZRAHI A., FAURE H. Enquête nationale sur le radiodiagnostic : Partie 3: Analyse comparative de l'activité des différents secteurs. J. Radiologie 66 (4) : 329-336; 1985.

[7] GOLDMAN L.W. Analysis of retakes: understanding, managing, and using an analysis of retakes program for quality assurance. HEW Publication (FDA) 79-8097 Washington, D.C. : U.S. Department of Health and Human Services, 1979.

[8] HALL C.L. Economic analysis from a quality control program. Pro. Soc. Photo-opt. Instrum. Eng., 1971, 127, 271-275.

[9] MACCIA C., BENEDITTINI M., LEFAURE C., FAGNANI F. Doses to patients from diagnostic radiology in France. Health Physics 54. No 4, 397-408, 1988.

[10] PADOVANI R., CONTENTO G., FABRETTO M., MALISAN M.R., BARBINA V., GOZZI G. Patient doses and risks from diagnostic radiology in North-East Italy, Br. J.'Radiol. 60 : 155-165, 1987.

[11] SHRIMPTON P.C., WALL B.F., JONES D.G., FISHER E.S., HILLIER M.C., KENDALL.G.M., HARRISSON R.M. A national survey of doses to patients undergoing a selection of routine X-ray examinations in English hospitals. NRPB-200 (HMSO, London), 1986.

[12] UNSCEAR. Rayonnements ionisants : niveaux et effets; publications des Nations Unies, Vol. 1 : Niveaux; Vol. 2 : Effets ; 1958, 1962, 1964, 1966, 1969, 1974, 1982, 1988. 


\section{ANNEXE \\ CRITĖRES D'IMAGE DU CLICHÉ DU THORAX (P/A).}

- Cliché pris en inspiration forcée.

- Reproduction symétrique du thorax.

- Reproduction de l'ensemble de la cage thoracique au-dessus du diaphragme.

- Reproduction de la trame vasculaire dans tout le poumon, en particulier les vaisseaux périphériques.

- Reproduction visuellement nette

a) de l'arbre bronchique, de la silhouette cardiaque et de l'aorte,

b) du diaphragme et des sinus costo-diaphragmatiques.

- Visualisation du poumon rétrocardiaque et du médiastin.

\section{CRITĖRES D'IMAGE DU CLICHÉ DU CRÂNE (PIA et A/P)}

- Reproduction symétrie du crâne, en particulier de la calotte, des orbites et des rochers.

- Projection de l'apex des rochers au centre des orbites.

- Reproduction visuellement nette du sinus frontal, des cellules ethmoïdales, de l'apex du temporal et des conduits auditifs internes.

- Reproduction visuellement nette des tables externe et interne de la voûte du crâne.

\section{CRITERES D'IMAGE DU CLICHÉ DU CRÂNE (profil)}

- Reproduction visuellement nette des tables externe et interne de la voûte du crâne, du plancher de la selle turcique et de l'apex des rochers du temporal.

- Superposition exacte respectivement des contours de la fosse crânienne antérieure, de la petite aile du sphenoïde, des apophyses clinoïdes des conduits auditifs externes.

- Reproduction visuellement nette des canaux vasculaires, du vertex et de la structure spongieuse du crâne.

\section{CRITERES D'IMAGE DU CLICHÉ DE LA COLONNE LOMBAIRE (A/P)}

- Reproduction linéaire des plateaux des corps supérieurs et inférieurs vertébraux dans la zone centrale du faisceau et visualisation des espaces intervertébraux.

- Reproduction visuellement nette des pédicules.

- Visualisation des articulations interapophysaires.

- Reproduction des apophyses épineuses et transverses.

- Reproduction visuellement nette des structures compactes et spongieuses.

- Reproduction des tissus mous adjacents.

\section{CRITĖRES D'IMAGE DU CLICHÉ DE LA COLONNE LOMBAIRE (profil)}

- Reproduction linéaire des plateaux des corps supérieurs et inférieurs vertébraux dans la zone centrale du faisceau et visualisation des espaces intervertébraux.

- Superposition totale des arêtes vertébrales postérieures.

- Reproduction de la racine des arcs neuraux.

- Reproduction des pédicules et des trous de conjugaison.

- Visualisation des articulations interapophysaires.

- Reproduction visuellement nette des structures compactes et spongieuses.

- Reproduction des tissus mous adjacents. 


\section{CRITĖRES D'IMAGE DE LA CHARNIĖRE LOMBO-SACRÉE (profil)}

- Reproduction en projection tangentielle du plateau du corps vertébral inférieur de L5 et du plateau du corps vertébral supérieur de $\mathrm{S} 1$.

- Visualisation de l'arête antérieure du sacrum supérieur.

- Reproduction des pièces vertèbres du sacrum supérieur.

\section{CRITĖRES D'IMAGE DU CLICHÉ DU BASSIN (A/P)}

- Reproduction symétrique du bassin.

- Visualisation du sacrum et de ses trous de conjugaison.

- Visualisation du pubis et de l'ischion.

- Reproduction des cols fémoraux qui ne doivent pas être déformés par raccourcissement ou par rotation.

- Reproduction de l'os spongieux et cortical et visualisation des trochanters.

\section{CRITĖRES D'IMAGE DU CLICHÉ DE L'APPAREIL URINAIRE}

\section{Avant injection de produit de contraste:}

- Reproduction de tout l'appareil urinaire du pôle supérieur des reins au plancher de la vessie.

- Reproduction des contours des reins.

- Visualisation des contours du muscle psoas.

- Reproduction visuellement nette des os.

\section{Après injection de produit de contraste:}

- Augmentation de la densité du parenchyme (effet néphrographique).

- Remplissage visuellement nette du bassinet et des calices (effet pyélographique).

- Reproduction de la jonction pyélo-urétérale.

- Visualisation de la région normalement traversée par les uretères.

- Reproduction de toute la vessie.

CRITĖRES D'IMAGE DU CLICHÉ DU SEIN (plusieurs incidences possibles)

- Reproduction visuellement nette du sein.

- Reproduction de la paroi thoracique adjacente.

- Reproduction visuellement nette de la peau et du tissu sous-cutané.

- Le mamelon doit être parallèle au film. 